\title{
Dissecting indirect genetic effects from peers in laboratory mice
}

Amelie Baud ${ }^{1,2,3^{*}}$ (D), Francesco Paolo Casale ${ }^{1,4}$, Amanda M. Barkley-Levenson², Nilgoun Farhadi ${ }^{2}$, Charlotte Montillot ${ }^{5}$, Binnaz Yalcin ${ }^{5}$, Jerome Nicod ${ }^{6,7}$, Abraham A. Palmer ${ }^{2,8}$ and Oliver Stegle $e^{1,9,10,11^{*}}$

\author{
* Correspondence: amelie.baud@ \\ crg.eu; oliver.stegle@embl.de \\ ${ }^{1}$ European Molecular Biology \\ Laboratory, European Bioinformatics \\ Institute, Wellcome Genome \\ Campus, CB10 1SD Hinxton, \\ Cambridge, UK \\ Full list of author information is \\ available at the end of the article
}

\begin{abstract}
Background: The phenotype of an individual can be affected not only by the individual's own genotypes, known as direct genetic effects (DGE), but also by genotypes of interacting partners, indirect genetic effects (IGE). IGE have been detected using polygenic models in multiple species, including laboratory mice and humans. However, the underlying mechanisms remain largely unknown. Genomewide association studies of IGE (igeGWAS) can point to IGE genes, but have not yet been applied to non-familial IGE arising from "peers" and affecting biomedical phenotypes. In addition, the extent to which igeGWAS will identify loci not identified by dgeGWAS remains an open question. Finally, findings from igeGWAS have not been confirmed by experimental manipulation.

Results: We leverage a dataset of 170 behavioral, physiological, and morphological phenotypes measured in 1812 genetically heterogeneous laboratory mice to study IGE arising between same-sex, adult, unrelated mice housed in the same cage. We develop and apply methods for igeGWAS in this context and identify 24 significant IGE loci for 17 phenotypes (FDR < 10\%). We observe no overlap between IGE loci and DGE loci for the same phenotype, which is consistent with the moderate genetic correlations between DGE and IGE for the same phenotype estimated using polygenic models. Finally, we fine-map seven significant IGE loci to individual genes and find supportive evidence in an experiment with a knockout model that Epha4 gives rise to IGE on stress-coping strategy and wound healing.
\end{abstract}

Conclusions: Our results demonstrate the potential for igeGWAS to identify IGE genes and shed light into the mechanisms of peer influence.

Keywords: Indirect genetic effects, Social genetic effects, Peer effects, Genotype to phenotype, Genome-wide association study

\section{Background}

The phenotype of an individual can be affected not only by the individual's own genotypes (direct genetic effects, DGE) but also by environmental factors, including the genotypes of other, interacting individuals (indirect genetic effects, IGE) [1-3] (Fig. 1a). IGE arise when the phenotype of a focal individual is influenced by heritable traits of

(c) The Author(s). 2021 Open Access This article is licensed under a Creative Commons Attribution 4.0 International License, which permits use, sharing, adaptation, distribution and reproduction in any medium or format, as long as you give appropriate credit to the original author(s) and the source, provide a link to the Creative Commons licence, and indicate if changes were made. The images or other third party material in this article are included in the article's Creative Commons licence, unless indicated otherwise in a credit line to the material. If material is not included in the article's Creative Commons licence and your intended use is not permitted by statutory regulation or exceeds the permitted use, you will need to obtain permission directly from the copyright holder. To view a copy of this licence, visit http://creativecommons.org/licenses/by/4.0/. The Creative Commons Public Domain Dedication waiver (http://creativecommons.org/publicdomain/zero/1.0/) applies to the data made available in this article, unless otherwise stated in a credit line to the data. 


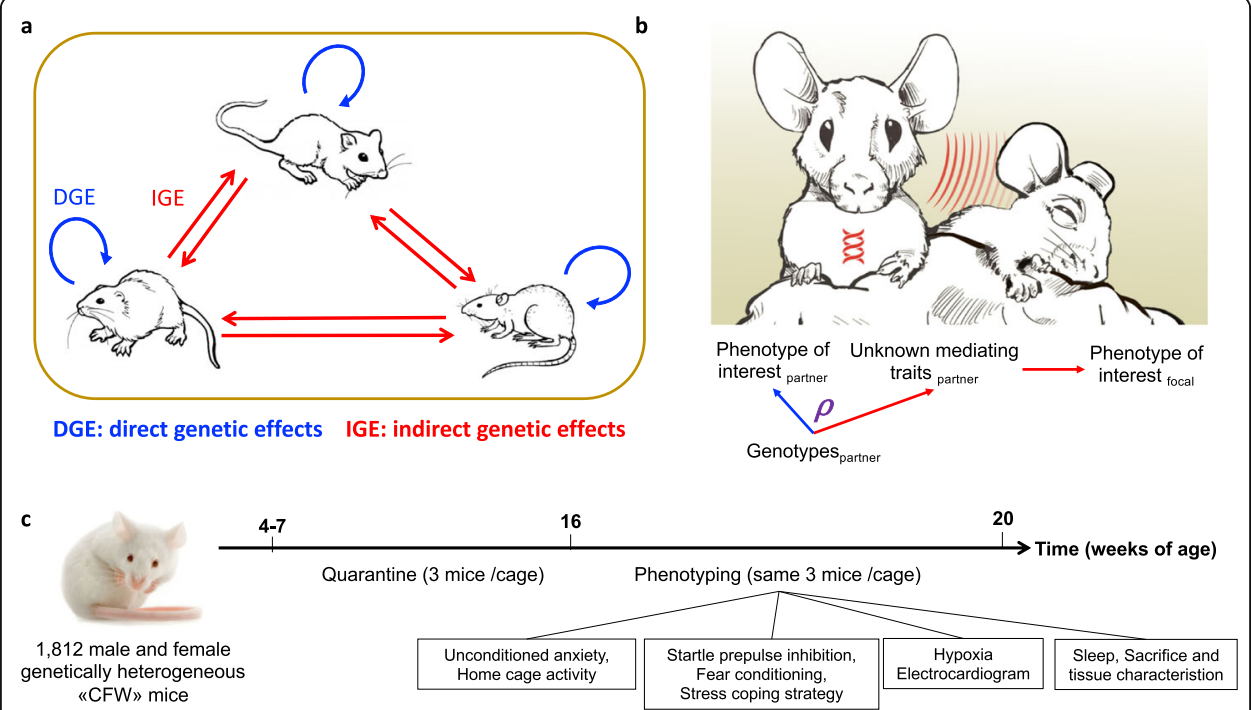

Fig. 1 Definition of direct and indirect genetic effects and experimental design. a Direct genetic effects (DGE, blue) on an individual's phenotype arise from the individual's own genotypes; indirect genetic effects (IGE, red) arise from genotypes of interacting partners (cage mates). This panel illustrates a situation where all individuals are genetically heterogeneous and both DGE and IGE arise from each individual's genotypes. b IGE on a phenotype of interest arise when two individuals interact and (unknown) heritable traits of one individual, the social partner, influence the phenotype of interest measured in the other individual, the focal individual. For a given phenotype of interest, the correlation $\rho$ between DGE and IGE is equivalent to the correlation between DGE on the phenotype of interest and DGE on the traits mediating IGE on the phenotype of interest. Importantly, this correlation can be estimated even when the traits mediating IGE are not known or not measured. c Experimental design. A list of the 170 phenotypes collected on each mouse is presented in Additional file 3: Table S2

interacting partners (Fig. 1b), which can include behavioral and non-behavioral traits of partners as well as modifications of the non-social environment by partners [4]. IGE have been detected in many laboratory systems [5-14], livestock [15-17], crops [18], wild animals [19-21], and humans [22-27], demonstrating that they are an important component of the genotype to phenotype path and an aspect of the environment that can be studied using genetic approaches.

Most prior studies of IGE have used polygenic modeling approaches to study aggregate genetic effects (i.e., sum of IGE across the genome), either focusing on IGE mediated by specific traits of partners using trait-based models [2, 28] or polygenic risk scores [22, 25], or detecting IGE mediated by unknown heritable traits of partners using variance component models $[9,15,29,30]$. More recently, the genome-wide association study of IGE (igeGWAS) has been proposed as a strategy to identify individual genetic loci underlying IGE $[5,7,8,11,31-35]$.

However, igeGWAS has only been applied in limited settings: in particular, it has not been used to study non-familial IGE from peers affecting biomedical phenotypes, despite growing evidence from polygenic models in laboratory mice [9] and in humans $[25,26]$ that such effects are important. Moreover, the relationship between DGE and IGE affecting the same phenotype has not been fully addressed, such that the scope for igeGWAS to identify loci not detected by dgeGWAS is unknown. Finally, the results of igeGWAS have not yet been translated into experimentally validated genes causing IGE. 
To address these issues, we leveraged a published dataset of 170 behavioral, physiological, and morphological phenotypes measured in 1812 male and female, genetically heterogeneous mice (Fig. 1c), which we supplemented with previously unreported cage information (Additional file 1: Table S1). For each phenotype, we investigated the relationship between DGE and IGE, using both polygenic analyses and GWAS. For 17 phenotypes, we fine-mapped IGE loci to identify putative causal genes underlying IGE. Finally, we tested one such gene using a knockout model.

\section{Results}

We used the genome-wide genotypes (both LD-pruned and unpruned genotypes derived from low-coverage (0.15x) Illumina sequencing, see the "Methods" section) and 200 phenotypes of 1934 commercially available, outbred Crl:CFW(SW)-US_P08(36) (hereafter CFW) mice reported in Nicod et al. [36] and Davies et al. [37]. In addition, we used previously unreported cage information provided by the authors of the original study upon request (Additional file 1: Table S1). Mice were housed in same-sex groups of three and interacted for at least 9 weeks before phenotyping. We excluded any animal whose cage mates changed over the course of the experiment, as well as suspected siblings to rule out confounding from parental and litter effects. These steps resulted in a final sample size of 1812 mice (927 females, 885 males) for analysis. We normalized each phenotype and excluded 30 phenotypes that could not be satisfactorily normalized (see the "Methods" section), yielding a total of 170 phenotypes measured in between 844 and 1729 mice.

\section{Polygenic analysis of the correlation between DGE and IGE}

Initially, we used polygenic models to assess the extent to which loci are shared between DGE and IGE affecting the same phenotype. Briefly, for each trait, we estimated the genetic correlation $\rho$ between DGE and IGE. As this correlation is equivalent to the correlation between DGE on the phenotype of interest and DGE on the traits mediating IGE (Fig. 1b), a correlation coefficient of 0 would indicate that the traits mediating IGE are genetically uncorrelated (in the classical sense) to the phenotype of interest, whereas a correlation coefficient of \pm 1 would indicate that the phenotype of interest itself mediates IGE. For 28 traits with evidence for marginal aggregate DGE and IGE (> $5 \%$ of phenotypic variance explained; Additional file 2: Fig. S1), we tested whether $\rho$ is equal to 0 and whether it is equal to \pm 1 (Fig. 2 and Additional file 3: Table S2). We found that $\rho$ is different from 0 for 10 out of 28 phenotypes $(\mathrm{P}<0.05)$, indicating that, often, the traits mediating IGE on a phenotype of interest are genetically correlated (in the classical sense) with the phenotype of interest. Evidence that $\rho$ is different from zero was strongest for mean weight of the adrenal glands, which correlates with stress [38], mean platelet volume, LDL cholesterol levels, and rate of healing from an ear punch. Second, $\rho$ is different from \pm 1 for 10 phenotypes $(\mathrm{P}<0.05)$, with the strongest evidence for a measure of stress-coping strategy (immobility in the forced swim test) and rate of healing from an ear punch. These results indicate that IGE on a phenotype of interest are often mediated by traits of partners different from the phenotype of interest. To uncover those traits, we turned to igeGWAS. 


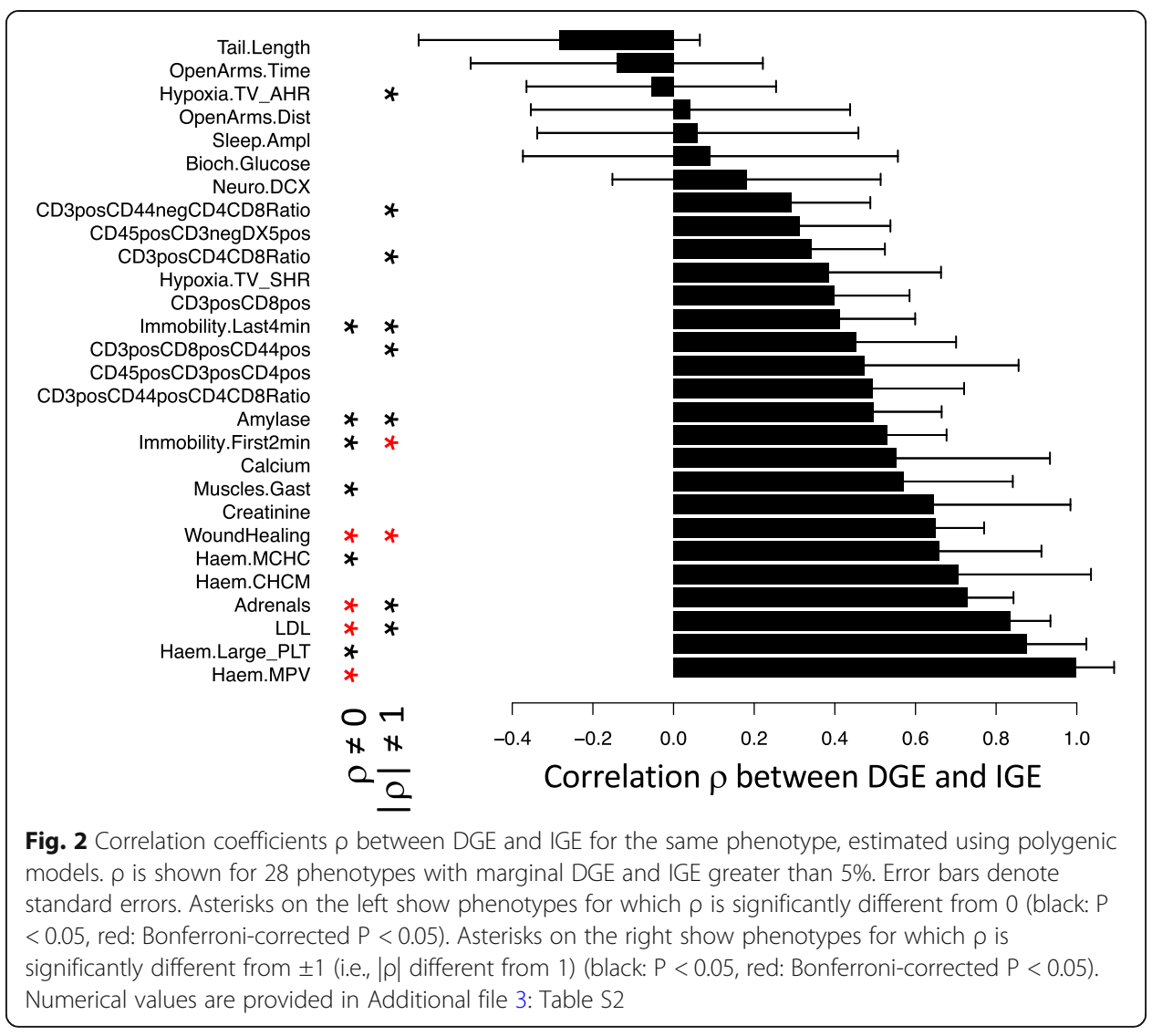

igeGWAS and dgeGWAS of the same 170 phenotypes

Next, to compare DGE and IGE at the level of individual loci, we considered the LDpruned set of variants and performed igeGWAS and dgeGWAS in an analogous manner for each of the 170 phenotypes. For igeGWAS, we estimated the "social genotype" of a mouse at a variant as the sum of the reference allele dosages across its two cage mates at the variant $[31,39]$ and tested for association between this social genotype and the phenotype of interest. To avoid spurious associations, we accounted for background IGE, background DGE, and shared environmental (cage) effects using random effect components in a linear mixed model (see the "Methods" section). Additionally, we included a fixed effect covariate for DGE arising from the tested variant in igeGWAS. This approach accounts for correlations between direct and social genotypes arising when each individual serves as both focal individual and social partner in the analysis, a strategy that maximizes sample size when all the individuals are genotyped and phenotyped [31, 39]. Accounting for such correlations was required to obtain appropriately calibrated P values in our cohort (Additional file 2: Fig. S2), and theoretical considerations show that it is required even when considering strictly unrelated samples (Additional file 2: Supplementary Note). Finally, we adapted previous strategies $[36,40,41]$ based on genome-wide permutations to control the per-phenotype FDR (see the "Methods" section), thereby accounting for the specific patterns of linkage disequilibrium present in the sample.

igeGWAS identified a total of 24 significant loci across 17 of the 170 tested phenotypes (FDR $<10 \%$ ), including measures relevant to behavior, adult neurogenesis, blood 
biochemistry, red and white blood cells, apparent bone mineral content, electrocardiography, and ventilatory responses to acute hypoxia (Additional file 4: Table S3). The 17 phenotypes with one or more significant IGE loci tended to have a higher aggregate contribution of IGE than phenotypes without significant IGE loci (averages of $3.8 \%$ and $2.8 \%$, respectively), a trend that was not significant (one-sided t-test $\mathrm{P}=0.14$ ).

To enable a direct comparison between igeGWAS and dgeGWAS, we performed dgeGWAS for each phenotype using the same approach as taken for igeGWAS, including random effects for DGE and IGE polygenic effects and cage effects and including a fixed effect covariate for IGE arising from the tested variant. This identified 120 significant DGE loci for 63 phenotypes (FDR < 10\%; Additional file 5: Table S4). Consistent with the difference in the number of discoveries, we observed that significant IGE loci had, on average, lower effect sizes (proportion of phenotypic variance explained) than DGE loci (Additional file 2: Fig. S3). In light of the observed effect sizes and due to the winner's curse (or Beavis effect $[42,43]$ ), we expect a larger proportion of significant IGE loci to be false associations compared to significant DGE loci.

There was no overlap between significant DGE and IGE loci for the same phenotype, or even for related phenotypes (Fig. 3, Additional file 4: Table S3 and Additional file 5: Table S4). This observation was expected based on the moderate values observed for the correlation $\rho$ between DGE and IGE and the limited power of dgeGWAS and igeGWAS. However, we identified further reason why dgeGWAS and igeGWAS might identify different loci: using simulations to identify key parameters determining the power of igeGWAS, we found that both the number of cage mates and the mode of aggregation across cage mates (i.e., whether the IGE received by a focal mouse correspond to the sum or the average of the IGE emitted by its cage mates) are important, in addition to the parameters also determining the power of dgeGWAS, namely minor allele frequency (MAF) and allelic effect (Additional file 2: Fig. S4). Thus, for a given MAF, allelic effect and a number of cage mates equal to two as is the case in this study, dgeGWAS is expected to have greater power than igeGWAS if IGE get averaged across the two cage mates, but igeGWAS is expected to have greater power than dgeGWAS if IGE sum up across the two cage mates. Importantly, the mode of aggregation across cage mates (sum or average) can be different for different phenotypes. As sample sizes increase for dgeGWAS and igeGWAS, the moderate genetic correlation $\rho$ between DGE and IGE and the differences in power between dgeGWAS and igeGWAS dictated by the number of cage mates and the mode of aggregation across cage mates will continue to drive the identification of different loci by dgeGWAS and igeGWAS.

\section{Identification of putative causal genes for experimental evaluation}

Linkage disequilibrium decays faster in the CFW population than in many other mouse populations used for mapping, which facilitates identification of putative causal genes at associated loci $[36,44,45]$. To identify such genes, we fine-mapped the 24 significant IGE loci using the full set of variants (rather than the pruned set used for igeGWAS) in the $1.5-\mathrm{Mb}$ window surrounding the most significant variant at the locus, which corresponds, in this sample, to the average 95\% confidence interval for the association [36]. We then identified, for each significant IGE locus, all the genes that either overlap the associated plateau or are located in direct proximity (see the "Methods" section, genes 


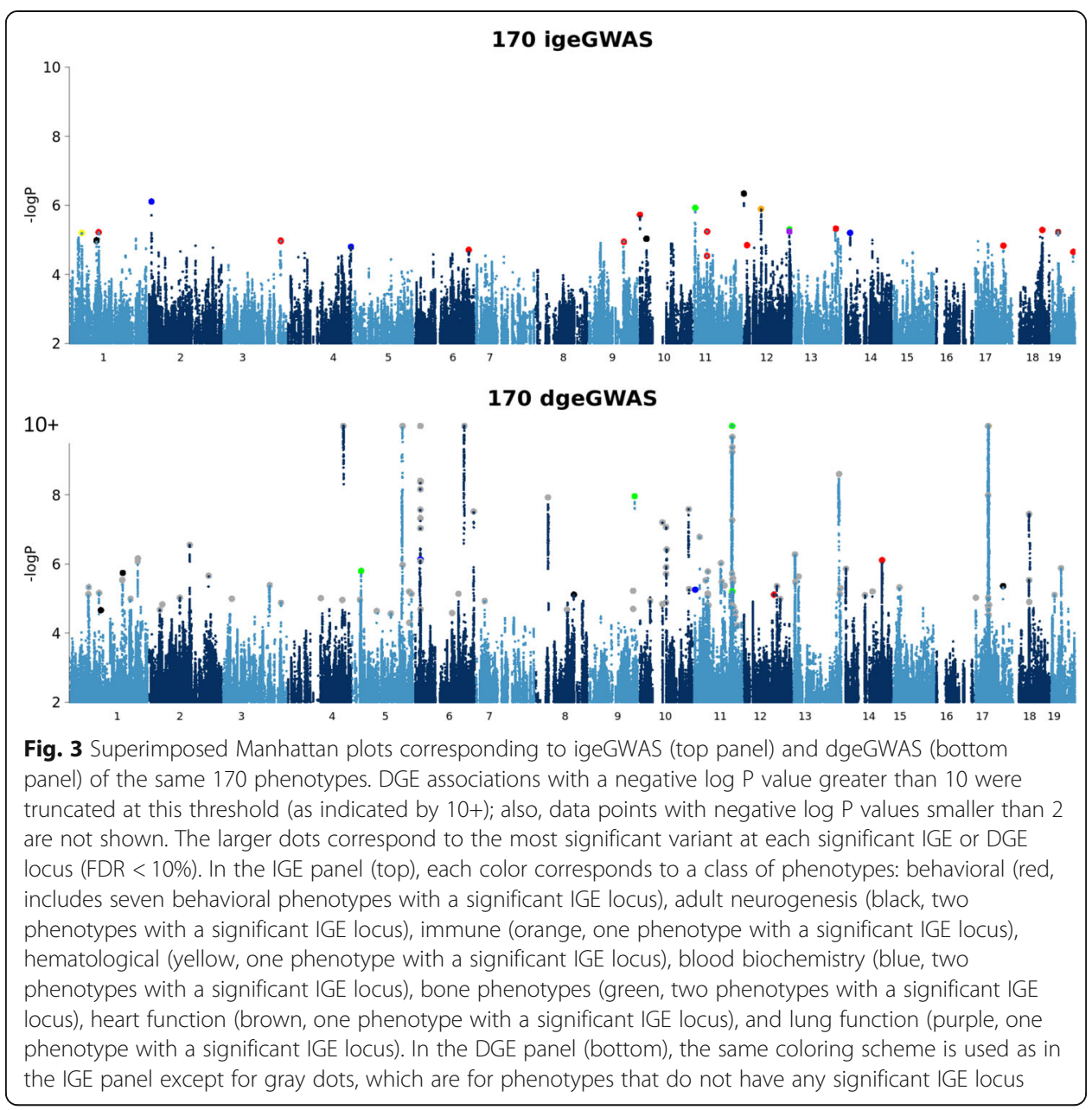

listed in Additional file 4: Table S3 and local association plots available from figshare [46]). At seven loci, there was a single putative causal gene: Abca12 at a locus for adult neurogenesis; Epha4 (stress-coping strategy); Pkn2, Slit3, and Pgk1-rs7 (at three different loci for sleep); H60c (home cage activity); and Adcyl (osteopetrosis).

One example of a putative causal IGE gene identified via this strategy is Epha4, which was identified at an IGE locus on chromosome 1 for immobility during the first 2 min of the forced swim test (FST), a measure of stress-coping strategy [47] (Fig. 4a and Additional file 2: Fig. S5). We focused on Epha4 initially because it was the only putative causal gene at a significant locus, the locus was in the top half of the list in terms of significance, and a knockout mouse model was readily available from a neighboring institute. In addition, Epha4 is in a gene desert and the only protein-coding gene in a chromatin topological domain [48, 49], meaning the causal variant(s) at the Epha4 locus likely affect the expression of Epha4 and not that of a neighboring gene.

Epha4 encodes a synaptic protein that plays an important role in synaptic plasticity in the hippocampus $[50,51]$ and DGE of Epha4 on FST immobility have been reported [52, 53]. Therefore, we evaluated the possibility that Epha4 directly influences stresscoping strategy and that the stress-coping strategy of a mouse in the weeks prior to or during the FST gets copied by the other mice in the cage (behavioral contagion), thereby giving rise to IGE on stress-coping strategy. To investigate this hypothesis, we 


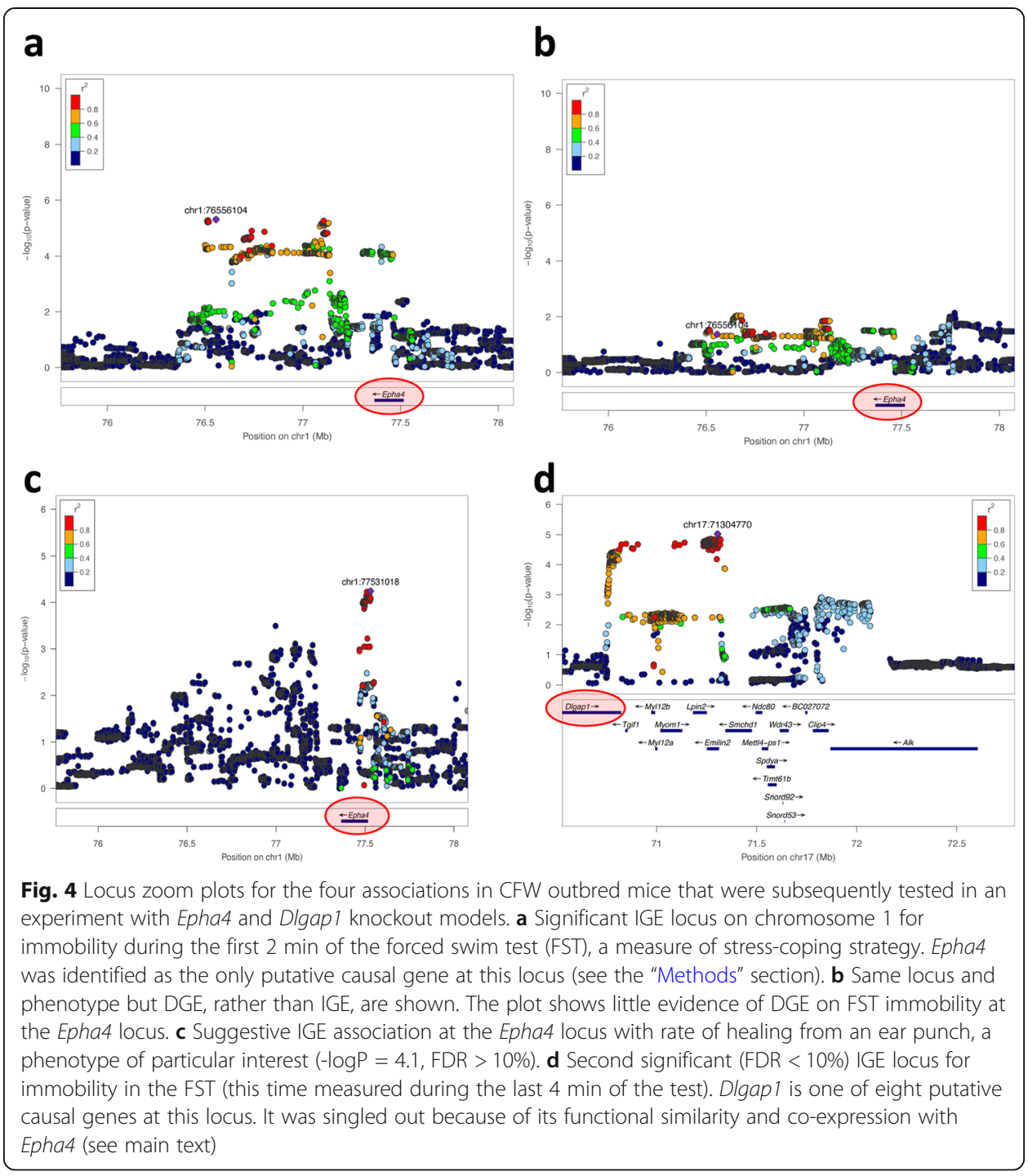

tested whether Epha4 has direct effects on FST immobility in CFW mice, using the full set of variants in the same $1.5-\mathrm{Mb}$ window including Epha4 as for IGE analysis. We found little evidence that Epha4 directly affects FST immobility in CFW mice (maximum - $\log \mathrm{P}$ value at the locus. 2.14, Fig. 4b), making it unlikely that behavioral contagion explains the detected IGE in CFW mice.

In addition to the significant IGE association between Epha4 and FST immobility, we found suggestive evidence for an IGE association between Epha4 and rate of healing from an ear punch (igeGWAS $-\log \mathrm{P}$ value $=4.1$, FDR $>10 \%$, Fig. 4c). This finding was of particular interest because the Epha4 locus was among the three most significant IGE loci for wound healing (all three loci with $-\log \mathrm{P}=4.1$, FDR $>10 \%$ ) and because IGE on wound healing seem to be ubiquitous in laboratory mice: indeed, we have found a significant aggregate contribution of IGE to rate of healing from an ear punch in all three mouse populations we have looked at to date (inbred C57BL/6 $\mathrm{J}$ mice and outbred Heterogeneous Stock mice in Baud et al. [9], and CFW mice in this study). Thus, we were particularly interested in testing whether Epha4 is involved in IGE on wound healing. 
We found two additional significant IGE loci for FST immobility, more precisely for immobility during the last $4 \mathrm{~min}$ of the test (Additional file 4: Table S3, Additional file 2: Fig. S6a). At the locus on chromosome 17, we identified eight genes as putatively causal but singled out Dlgap1 (Fig. 4d) for experimental validation because it encodes a synaptic protein [54], like Epha4, and because its expression in the hippocampus, which was measured in a separate cohort of 79 male CFW mice [45], is significantly and highly correlated with that of Epha4 (Spearman $\mathrm{r}=0.868$, Bonferroni-corrected $\mathrm{P}=$ 2.3.10 ${ }^{-19}$, Additional file 2: Fig. S6b). As was the case for Epha4, we found no evidence of DGE arising from Dlgap1 and affecting FST immobility in CFW mice (maximum $-\log \mathrm{P}$ value at the locus 2.46$)$.

\section{Evaluating the role of Epha4 and Dlgap1 in IGE using knockout models}

We tested the hypotheses that Epha4 can give rise to IGE on FST immobility and rate of healing using a constitutive Epha4 knockout model on a mixed C57BL/6, C57BL/ 10 and 129 genetic background. In addition, we tested for IGE from Dlgap1 on FST immobility using a constitutive Dlgap1 knockout model on a C57BL/6N background. At weaning, one Epha4 mouse (heterozygote or wild-type, see the "Methods" section) or one Dlgap1 mouse (homozygote knockout, heterozygote, or wild-type) was co-housed with one focal FVB/NJ (FVB) mouse of the same sex (male or female). The FVB strain was chosen because it is the inbred strain whose genetic background is most similar to that of the outbred CFW mice used in igeGWAS, contributing $38 \%$ of all alleles in CFW mice [36]. Focal FVB mice were ear punched prior to pairing, then the pairs of mice were left to interact in their cages for two months before they were all tested in the FST and the ears of FVB mice were analyzed to measure the rate of healing (see the "Methods" section).

Although FVB mice are genetically similar to CFW mice, we observed that focal FVB mice showed much less immobility during the first 2 min of the FST than CFW mice ( $2.0 \mathrm{~s}$ on average across all FVB mice vs $12.2 \mathrm{~s}$ on average across all CFW mice). Therefore, in our analysis of FVB focal mice, we focused on immobility during the last $4 \mathrm{~min}$ of the test, even though this measure showed a less significant association in igeGWAS than immobility during the first $2 \mathrm{~min}$ of the test ( $\log \mathrm{P}=2.8$ and 5.2 , respectively).

When considering males and females together, we found no effect of the genotype of cage mates on either FST immobility ( $\mathrm{P}=0.52$, ANOVA, $\mathrm{N}=81$ ) or wound healing ( $\mathrm{P}$ $=0.40$, ANOVA, $\mathrm{N}=85$ ). However, model comparison using the Akaike Information Criterion (AIC) indicated there is an interaction between sex and genotype of the cage mate (i.e., IGE) for both FST immobility and wound healing, as the model including an interaction term between sex and genotype of the cage mate was favored. Therefore, we considered the two sexes separately and observed, in males but not in females, suggestive evidence of IGE on FST immobility ( $\mathrm{P}=0.054$, ANOVA, $\mathrm{N}=35)$ and wound healing ( $\mathrm{P}=0.038$, ANOVA, $\mathrm{N}=38$ ) (Fig. 5). The detection of male-specific IGE from Epha4 on wound healing is consistent with the observation of stronger IGE at the Epha4 locus in male CFW mice compared to female CFW mice (Additional file 2: Fig. S7a). The detection of male-specific IGE on FST immobility, on the other hand, was not expected from the analysis of CFW mice as similar effects were observed in males and females (Additional file 2: Fig. S7b and S7c). A potential explanation for male- 
specific IGE on FST immobility in FVB focal mice is that FVB females show lower immobility than FVB males (Fig. 5), hindering our ability to detect genetic effects. Nevertheless, these experimental results support the hypotheses made following igeGWAS that Epha4 can give rise to IGE on FST immobility and wound healing in laboratory mice.

As was the case in CFW mice, we did not observe a direct effect of Epha4 on FST immobility whether all mice or males only were considered $(\mathrm{P}=0.22$ and 0.23 , respectively, ANOVA, $\mathrm{N}=81$ and 35 , respectively), indicating behavioral contagion is unlikely to explain these IGE.

Finally, we found no evidence of IGE from Dlgap1 on FST immobility.

\section{Discussion}

In this study, we leveraged a published dataset of 170 behavioral, physiological, and morphological phenotypes measured in 1812 genetically heterogeneous mice housed in same-sex groups of three to characterize the relationship between DGE and IGE for the same phenotype and identify individual IGE loci. Using polygenic models, we showed that the genetic correlation $\rho$ between DGE and IGE for the same phenotype is often significantly different from \pm 1 , indicating IGE loci are different from DGE loci for the same phenotype. Consistently, we found that none of the 24 significant IGE loci identified for 17 phenotypes using igeGWAS overlapped with significant DGE loci identified for the same phenotypes using dgeGWAS. We fine-mapped seven significant IGE loci to a single putative causal gene and experimentally validated IGE from one of them, Epha4, on stress-coping strategy and wound healing using a knockout model.

The analysis of the genetic correlation $\rho$ between DGE and IGE for the same phenotype provides insights into the overlap between DGE and IGE loci for a given phenotype and whether the traits mediating IGE on a phenotype of interest are genetically correlated (in the classical sense) with that phenotype. The correlation $\rho$ was expected

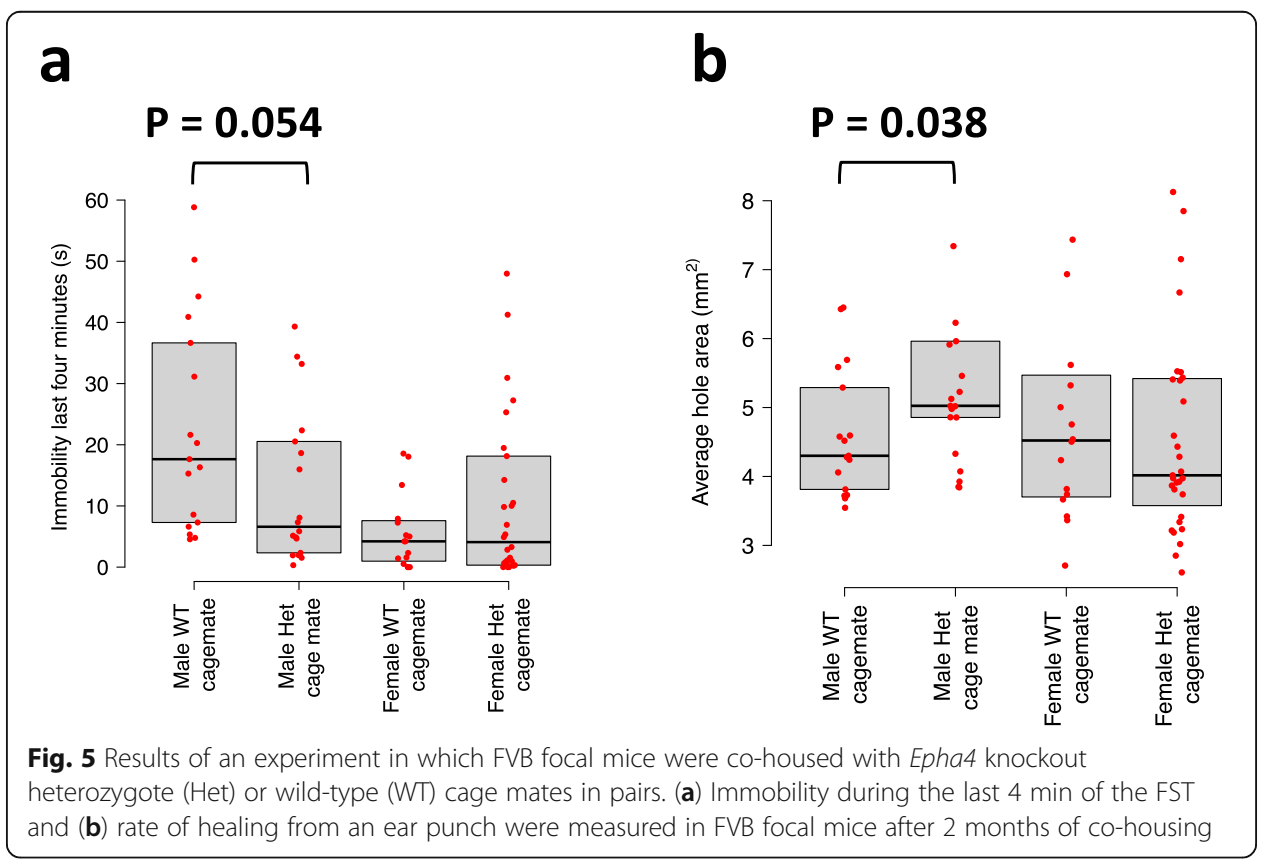


to be different from 0 for many phenotypes, based on reports that emotions [55-57], behaviors [25, 58, 59], pathogens, and components of the gut microbiome [60] can "spread" between individuals and contribute to phenotypic variation, both in mice and in humans. In our study, we found that $\rho$ is significantly different from 0 for a variety of phenotypes, which indicates some overlap between DGE loci and IGE loci for the same trait and is consistent with a genetic correlation (in the classical sense) between the phenotype of interest and the traits mediating IGE. However, we also found that $\rho$ is significantly different from \pm 1 for 10 out of 28 traits, reflecting differences between DGE and IGE loci and demonstrating that IGE on a phenotype of interest often involve traits of cage mates other than the phenotype of interest. This was true even for phenotypes that spread, namely stress and stress-coping strategies. Notably, a similar conclusion has been reached in a study of IGE between spouses in the UK BioBank [26].

Consistent with the estimates of $\rho$ from polygenic models, we found no overlap between the 24 loci identified by igeGWAS for 17 phenotypes and the loci identified by dgeGWAS for the same phenotypes. Our survey of a large number of phenotypes suggests that the loci identified by igeGWAS will, generally, be different from those identified by dgeGWAS, meaning igeGWAS holds great potential to uncover new loci underlying phenotypic variation and that these loci will point to traits of cage mates different from the phenotype studied.

Identifying IGE genes using igeGWAS has been previously attempted $[5,7,8,11,31$ 35], but there has been limited evidence that this approach can indeed identify genes that are causally involved in IGE. The results of our igeGWAS and fine-mapping analyses identified a single putative causal gene at seven IGE loci: Abca12 at a locus for adult neurogenesis; Epha4 at a locus for stress-coping strategy; Pkn2, Slit3, and Pgk1rs7 at three different loci for sleep; H60c at a locus for home cage activity; and Adcy1 at a locus for osteopetrosis. Among the loci with a single putative causal gene, the Epha4 locus stood out because of its relatively high significance and because Epha4 is the only gene in a well-characterized topological domain $[48,49]$, meaning the causal variant(s) at the Epha4 locus likely affect the expression of Epha4 and not that of a neighboring gene. Hence, we tested Epha4 in an experiment with a knockout model. Model selection analysis using the AIC indicated an interaction between the Epha4 allele and the sex of cage mates for both stress-coping strategy and wound healing and, indeed, we found suggestive evidence of IGE from Epha4 on both phenotypes in male mice ( $\mathrm{P}=$ 0.054 and 0.038 , respectively), but not in females. A limitation of our experiment is that FVB focal mice showed little to no immobility during the first 2 min of the FST, in contrast with the CFW mice used in igeGWAS. Hence, even though the significant igeGWAS locus was for immobility during the first $2 \mathrm{~min}$ of the test, we had to focus on immobility during the last 4 min when analyzing the behavior of FVB mice. Similarly, immobility during the last 4 min was lower in FVB female mice than FVB male mice, which may explain why we only observed IGE from Epha4 in males. Effects of the genetic background of knockout models have been reported in studies of DGE [61]; our results show that in studies of IGE the genetic background of the focal individuals matters too. In the future, we will consider a broader range of genetic backgrounds for focal mice.

The seven genes listed above as single putative causal genes at IGE loci are valuable starting points to gain insights into the mechanisms of IGE. In some cases, it is possible 
to make testable hypotheses as to the mechanisms mediating IGE from knowledge of the expression profile and function of the gene. For example, Epha4 plays an important role in the development of the central nervous system and in synaptic plasticity [50,51], and it has been reported to have DGE on stress-coping strategy [52, 53]. Hence, we hypothesized that IGE from Epha4 were mediated by behaviours of cage mates, more specifically by focal mice copying the stress-coping strategy of their cage mates (behavioral contagion). We found no evidence of DGE from Epha4 in either CFW or FVB mice however, suggesting other behaviours of cage mates-or other traits altogether-are involved. As far as wound healing is concerned, the reported expression of Epha4 in platelets in humans [62], the presence of platelets in saliva [63], and the role of platelets in wound healing and tissue regeneration [64] suggest wound licking by cage mates may be mediating IGE from Epha4 on wound healing.

Finally, we identified challenges and solutions to different sources of confounding in igeGWAS. In particular, we demonstrated that correlations between direct and social genotypes arise when study individuals play both roles of focal individuals and social partners and that, counter-intuitively, these correlations arise even when all individuals are strictly unrelated. We showed that accounting for direct effects of the locus tested in the null model for igeGWAS permits avoiding spurious IGE associations. These insights, combined with the light we shed on two key parameters determining the power of igeGWAS, namely the number of cage mates and the mode of aggregation of IGE across cage mates, will inform the design and analysis of future igeGWAS.

\section{Conclusions}

Our results demonstrate the potential for igeGWAS to uncover genetic effects expressed only in the context of social interactions and to serve as a starting point for follow-up analyses and experiments that will improve our understanding of peer effects on health and disease.

\section{Methods}

\section{Phenotypes and experimental variables}

Phenotypes and experimental variables (covariates) for 1934 male and female $\mathrm{Crl}$ : CFW(SW)-US_P08 (CFW) mice were retrieved from http://wp.cs.ucl.ac.uk/ outbredmice/. Phenotypes were normalized using the boxcox function (MASS package [65]) in R; phenotypes that could not be normalized satisfactorily (transformation parameter lambda outside of -2 to 2 interval) were excluded. Because data for some phenotypes were missing for some mice, the sample size varied. The sample size for each phenotype after all filtering (see below) is indicated in Additional file 3: Table S2. The subset of covariates used for each phenotype, which always included sex, is indicated in Additional file 3: Table S2. For those phenotypes where body weight was included as a covariate, we checked that this did not lead to systematically increased (or decreased) estimates of the aggregate contribution of IGE (collider bias).

\section{Cage information}

Mice were 4 to 7 weeks old when they arrived at the phenotyping facility and were housed in same-sex groups of three mice. They were left undisturbed for 9 to 12 weeks 
during their time in quarantine and spent another 4 weeks together during phenotyping.

Cage assignments were not included in the publicly available dataset but were provided by the authors upon request and are now provided in Additional file 1: Table S1. Cage assignments were recorded at eleven time points throughout the study and showed that a few mice were taken out of their original cages and singly housed, presumably because they were too aggressive. We only included in our analyses mice that had the same two cage mates throughout the experiment. We further excluded a subset of mice based on their genotype-based genetic similarity, as described below.

Finally, all mice were singly housed during the sleep test and until sacrifice a few days later. Hence, we investigated "persistent" IGE on sleep and tissue phenotypes.

\section{Genome-wide genotypes}

From http://wp.cs.ucl.ac.uk/outbredmice/, we retrieved both allele dosages for 7 million variants and allele dosages for a subset of 353,697 high quality, LD-pruned variants (as described in Nicod et al. [37]; genotyping based on sparse $0.15 x$ sequencing data). We used LD-pruned variants for all analyses but the identification of putative causal genes at IGE loci (see below), for which we used the full set of variants.

\section{Genetic relatedness matrix and exclusion of presumed siblings}

The genetic relatedness matrix (GRM) was calculated as the cross-product of the LDpruned dosage matrix after standardizing the dosages for each variant to mean 0 and variance 1. A few pairs of mice were outliers in the distribution of GRM values, which made us suspect that siblings had been included in the sample even though they were not supposed to be (siblings were excluded by design). To mitigate confounding of DGE and IGE analyses by parental and litter effects, we excluded 19 cages (57 mice) from all analyses.

\section{Variance component model}

The same model as described in detail in Baud et al. [9] was used. Briefly, the model used is the following:

$$
y_{f}=X_{f} \underline{b}+a_{D, f}+e_{D, f}+Z_{f} \underline{a_{S}}+Z_{f} \underline{e_{S}}+W_{f \underline{c}}
$$

$y_{f}$ is the phenotypic value of the focal mouse $f, X_{f}$ is a row of the matrix $X$ of covariate values, and $b$ a column vector of corresponding coefficients. $a_{D, f}$ is the additive direct genetic effects (DGE) of $f . Z_{f}$ is a row of the matrix $Z$ that indicates cage mates (importantly $Z_{i, i}=0$ ) and $\underline{a}_{S}$ the column vector of additive indirect (social) genetic effects (IGE). $\underline{e_{D}}$ refers to direct environmental effects (DEE) and $\underline{e_{S}}$ to indirect (social) environmental effects (IEE). $W_{f}$ is a row of the matrix $W$ that indicates cage assignment and $c$ the column vector of cage effects.

The joint distribution of all random effects is defined as: 


$$
\left[\begin{array}{c}
\frac{a_{D}}{a_{S}} \\
\frac{e_{D}}{e_{S}} \\
\underline{c}
\end{array}\right] \sim \operatorname{MVN}\left(0,\left[\begin{array}{ccccc}
\sigma_{A_{D}}^{2} \mathrm{~A} & \sigma_{A_{D S}} \mathrm{~A} & 0 & 0 & 0 \\
\sigma_{A_{D S}} \mathrm{~A}^{T} & \sigma_{A_{S}}^{2} \mathrm{~A} & 0 & 0 & 0 \\
0 & 0 & \sigma_{E_{D}}^{2} \mathrm{I} & \sigma_{E_{D S}} \mathrm{I} & 0 \\
0 & 0 & \sigma_{E_{D S}} \mathrm{I}^{T} & \sigma_{E_{S}}^{2} \mathrm{I} & 0 \\
0 & 0 & 0 & 0 & \sigma_{C}^{2} \mathrm{I}
\end{array}\right]\right.
$$

where A is the GRM matrix and I the identity matrix.

The phenotypic covariance is:

$$
\begin{gathered}
C_{i, j}=\operatorname{cov}\left(y_{i}, y_{j}\right)=\sigma_{A_{D}}^{2} A_{i, j}+\sigma_{A_{D S}}\left\{\left(A Z^{T}\right)_{i, j}+\left(Z A^{T}\right)_{i, j}\right\}+\sigma_{A_{S}}^{2}\left(Z A Z^{T}\right)_{i, j} \\
+\sigma_{E_{D}}^{2} I_{i, j}+\sigma_{E_{D S}}\left\{\left(I Z^{T}\right)_{i, j}+\left(Z I^{T}\right)_{i, j}\right\}+\sigma_{E_{S}}^{2}\left(Z I Z^{T}\right)_{i, j} \\
+\sigma_{C}^{2}\left(W I W^{T}\right)_{i, j}
\end{gathered}
$$

When all cages have the same number of mice, as is the case in this study, the nongenetic random effects are not identifiable $[15,66]$. An equivalent model can, in that case, be defined as [66]:

$$
\begin{array}{cc}
\operatorname{cov}\left(e_{i}, e_{j}\right)=\sigma_{E}^{2}=\sigma_{E_{D}}^{2}+2 \sigma_{E_{S}}^{2}+\sigma_{C}^{2} & \text { if } i=j \\
\operatorname{cov}\left(e_{i}, e_{j}\right)=\rho_{E} \sigma_{E}^{2}=2 \sigma_{E_{D S}}+\sigma_{E_{S}}^{2}+\sigma_{C}^{2} & \text { if } i \neq j \text { and } i \text { and } j \text { share a cage } \\
\operatorname{cov}\left(e_{i}, e_{j}\right)=0 & \text { if } i \text { and } j \text { are in different cages }
\end{array}
$$

We checked that both model (1) and this alternative model yielded the same genetic estimates and maximum likelihoods. The alternative model was fitted using the SimplifNonIdableEnvs option.

\section{Aggregate contributions of DGE and IGE}

The aggregate contributions of DGE and IGE were calculated, respectively, as sampleVar $\left(\sigma_{A_{D}}^{2} A\right) /$ sampleVar $(C)$ and sampleVar $\left(\sigma_{A_{S}}^{2}\left(Z A Z^{T}\right)\right) /$ sampleVar $(C)$,

where sampleVar is the sample variance of the corresponding covariance matrix: suppose that we have a vector $\underline{x}$ of random variables with covariance matrix $M$, the sample variance of $M$ is calculated as

$$
\text { sampleVar }(M)=\frac{\operatorname{Tr}(P M P)}{n-1}
$$

$\operatorname{Tr}$ denotes the trace, $n$ is the sample size, and $P=I-\frac{11^{\prime}}{n}$.

The significance of the IGE variance component was assessed using a two-degree of freedom log likelihood ratio (LLR) test (for the variance component and the covariance with DGE). Note that this testing procedure is conservative. The $\mathrm{Q}$ value for the aggregate contribution of IGE was calculated for each phenotype using the R package qvalue [67]. Significant IGE contributions were reported at FDR $<10 \%$ (corresponding to $\mathrm{Q}$ value $<0.1$ ).

\section{Correlation between DGE and IGE}

The correlation $\rho$ between $\underline{a_{D}}$ and $\underline{a_{S}}$ was calculated as:

$$
\rho=\frac{\sigma_{A_{D S}}}{\sigma_{A_{D}} \times \sigma_{A_{S}}}
$$

We tested whether $\rho$ was significantly different from 0 and whether $|\rho|$ was significantly different from 1 using a one-degree of freedom LLR test, which is conservative for the latter test. 


\section{Simulations for Additional file 2: Fig. S1}

Phenotypes were simulated based on the genotypes and cage relationships of the full set of 1812 mice. Phenotypes were drawn from model (1) with the following parameters: IGE explaining between 0 and $35.7 \%$ of phenotypic variance, DGE explaining $15 \%$ of phenotypic variance, $\rho_{A_{D S}}=0.47$, DEE explaining $22 \%$ of phenotypic variance, IEE explaining $16 \%$ of phenotypic variance, $\rho_{E_{D S}}=-0.97$, and cage effects explaining $26 \%$ of phenotypic variance. These variances correspond to the median value of estimates across traits with aggregate IGE and DGE $>5 \%$. After building the phenotypic covariance matrix, the sample variance of the simulations was calculated and used to calculate "realised" simulation parameters from the "target" parameters above. The realised parameters were used for comparison with the parameters estimated from the simulations.

\section{Models used for igeGWAS and dgeGWAS}

To test IGE of a particular variant in igeGWAS, we compared the following two models:

$$
\begin{aligned}
& y_{f}=X_{f} \underline{b}+a_{D, f}+e_{D, f}+Z_{f} \underline{a_{S}}+Z_{f} \underline{e_{S}}+W_{f} \underline{c}+G_{f} b_{D} \\
& y_{f}=X_{f} \underline{b}+a_{D, f}+e_{D, f}+Z_{f} \underline{a_{S}}+Z_{f} \underline{e_{S}}+W_{f \underline{c}}+G_{f} b_{D}+Z_{f} G b_{S}
\end{aligned}
$$

Here, $G$ is the vector of direct genotypes at the tested variant; hence, $G_{f}$ is the genotype of the individual that is phenotyped $(f)$ and $Z_{f} G$ is the sum of the genotypes of the two cage mates of $f$, the "social genotype" of $f[31,39]$. $b_{D}$ the coefficient for local DGE and $b_{S}$ the coefficient for local IGE. Note that $Z_{f}$ could be defined as the average of the genotypes of the two cage mates of $f$, in which case $\underline{b_{S}}$ would be doubled but the igeGWAS P values would remain unchanged. In igeGWAS, we refer to the inclusion of $G_{f} b_{D}$ in models (2) and (3) as "conditioning."

The models were fitted with the covariance of the model estimated only once per phenotype, in the model with no local genetic effect (model (1)).

The significance of local IGE was calculated by comparing models (2) and (3) with a 1-degree of freedom LLR test.

dgeGWAS was carried out by comparing model (3) above to the null model (4) below:

$$
y_{f}=X_{f} \underline{b}+a_{D, f}+e_{D, f}+Z_{f} \underline{a_{S}}+Z_{f} \underline{e_{S}}+W_{f} \underline{c}+Z_{f} G b_{S}
$$

In dgeGWAS, we refer to the inclusion of $Z_{f} G b_{S}$ in model (4) and (3) as "conditioning."

\section{Identification of significant associations}

We used a genome-wide permutation strategy to control the FDR for each phenotype, as done by Nicod et al. [36]. This strategy takes into account the specific patterns of linkage disequilibrium present in the sample and identifies significant associations for 
each phenotype independently of the results for the other phenotypes in the dataset. More precisely, for each phenotype and for each type of genetic effect (direct and indirect), we performed 100 "permuted GWAS" by permuting the rows of the matrix of social (respectively direct) genotypes and testing each variant at a time using the permuted genotypes together with the un-permuted phenotypes, un-permuted covariates, un-permuted GRM, and un-permuted matrix of direct (respectively social) genotypes (for conditioning) [40, 41]. For a given $P$ value $x$, the per-phenotype FDR can be calculated as:

$$
\operatorname{FDR}(x)=\frac{\# \text { loci with } P<x \text { in permuted data }}{100 \times \text { \#loci with } P<x \text { in unpermuted data }}
$$

We reported those loci with FDR $<10 \%$.

\section{Definition of putative causal genes at associated loci}

At each significantly associated locus, we defined a $1.5-\mathrm{Mb}$ window centered on the lead variant corresponding, in this sample, to the $95 \%$ confidence interval for the association [36]. We identified all the variants that segregate in this window based on the full set of 7M variants and reran igeGWAS and dgeGWAS locally using all the variants at the locus. We defined "putative causal genes" as those genes that either overlapped the associated plateau or were located in direct proximity, and whose MGI symbol does not start by "Gm," "Rik," "Mir," "Fam," or "Tmem" in order to focus on genes with known function and generate more tractable hypotheses on the mechanisms of IGE.

We identified putative causal genes using locusZoom plots [68]. To create them, we used the standalone version of locusZoom (https://genome.sph.umich.edu/wiki/ LocusZoom_Standalone). The plots for all 24 significant IGE loci reported in Additional file 4: Table S3 are available from figshare [46].

\section{Gene expression in the hippocampus of an independent sample of CFW mice}

Gene expression in the hippocampus of an independent sample of 79 male CFW mice, initially published in Parker et al. [45], was available from GeneNetwork (http:// genenetwork.org/) ([69, 70], (https://pubmed.ncbi.nlm.nih.gov/33472826/)). The data are accessible by selecting Mouse as Species, CFW Outbred GWAS as Group, Hippocampus mRNA as Type, and UCSD CFW Hippocampus (Jan17) RNA-Seq Log2 Zscore as Dataset. To retrieve the genes whose expression is most highly correlated with that of Epha4, we entered "Epha4" in the Get Any field. Following the selection of the Epha4 record (click on ENSMUSG00000026235), we used Calculate Correlations with Sample $\mathrm{r}$ as Method, UCSD CFW Hippocampus (Jan17) RNA-Seq Log2 Z-score as Database, and Spearman rank as correlation Type. Additional file 2: Fig. S6b is obtained by clicking on the value of the correlation between Epha4 and Dlgap1 expression levels (column Sample rho).

\section{Variance explained by a significant association}

The variance explained by a significant IGE association was estimated in an extension of model (1) with additional fixed effects for both DGE and IGE of lead SNPs at all significant IGE loci (the lead SNP being the SNP with the most significant P value at the locus in the igeGWAS). After fitting the model, the variance was calculated as: 


$$
\frac{\operatorname{var}\left(Z G \widehat{b_{S}}\right)}{\sum \operatorname{var}\left(X_{c} \widehat{b_{c}}\right)+\sum \operatorname{var}\left(G \widehat{b_{D}}\right)+\sum \operatorname{var}\left(Z G \widehat{b_{S}}\right)+\operatorname{sampleVar}(C)}
$$

where sampleVar $(C)$ is the sample variance of the covariance matrix in this model.

The variance explained by a significant DGE association was estimated in a similar model but considering all significant DGE associations and calculated as:

$$
\frac{\operatorname{var}\left(Z G \widehat{b_{D}}\right)}{\sum \operatorname{var}\left(X_{c} \widehat{b_{c}}\right)+\sum \operatorname{var}\left(G \widehat{b_{D}}\right)+\sum \operatorname{var}\left(Z G \widehat{b_{S}}\right)+\operatorname{sampleVar}(C)}
$$

\section{Simulations for Additional file 2: Fig. S2b and S2c}

Phenotypes were simulated based on the genotypes and cage relationships of the 1812 mice. Null phenotypes (no local IGE) were simulated from model (2) as the sum of random effects and local DGE. The following parameters were used for the random effects: $\sigma_{A_{D}}^{2}=20$ and $\sigma_{A_{S}}^{2}=20$ (which correspond to high polygenic effects in the real data), $\rho_{A_{D S}}=0.5, \sigma_{E_{D}}^{2}=30, \sigma_{E_{S}}^{2}=30, \rho_{E_{D S}}=-0.97$, and $\sigma_{C}^{2}=25$ (which are close to the median of the corresponding estimates from the real data). Local DGE were simulated at random variants in the genome to account for $20 \%$ of the phenotypic variance.

\section{Simulations for Additional file 2: Fig. S4}

Phenotypes were simulated based on the real genotypes but random cages. Phenotypes were simulated as the sum of random and fixed effects using the following models:

$$
\begin{aligned}
& y_{f}=X_{f} \underline{b}+a_{D, f}+e_{D, f}+Z_{f} a_{\underline{S}}+Z_{f} e_{\underline{S}}+W_{f} \underline{c}+G_{f} b_{D} \text { for local DGE } \\
& y_{f}=X_{f} \underline{b}+a_{D, f}+e_{D, f}+Z_{f} a_{\underline{S}}+Z_{f} e_{\underline{S}}+W_{f}+Z_{f} G b_{S} \text { for local IGE }
\end{aligned}
$$

The following parameter values were used for the random effects: $\sigma_{A_{D}}^{2}=17, \sigma_{A_{S}}^{2}=17$, $\rho_{A_{D S}}=0.65, \sigma_{E_{D}}^{2}=19, \sigma_{E_{S}}^{2}=15, \rho_{E_{D S}}=-0.8$, and $\sigma_{C}^{2}=25$. These values correspond to the median estimates for phenotypes with aggregate IGE and DGE $>0.1$.

Local DGE and IGE were simulated at variants with low MAF $(\mathrm{MAF}<0.05)$, medium MAF $(0.225<$ MAF $<0.275)$, or high MAF (MAF >0.45). Local IGE were simulated using two alternative generative models: an "additive" model by using $Z$ as in model (3) (i.e., filled with 0 s and 1s) or an "average" model by using $Z^{\prime}=\frac{Z}{N}$, where $N=2$. In all cases (DGE, additive IGE, and average IGE), we simulated an allelic effect of 0.2 , which is similar to the average allelic effect estimated in the igeGWAS. Power was calculated at a genome-wide significance threshold of negative $\log \mathrm{P} 5$, which is similar to the significance of associations detected at FDR $<10 \%$.

\section{Experiment with Epha4 and Dlgap1 knockout mice}

\section{Experimental design}

All animal procedures were approved by the Institutional Animal Care and Use Committee of the University of California San Diego (UCSD) and were conducted in accordance with the NIH Guide for the Care and Use of Laboratory Animals. FVB/NJ (hereafter FVB) breeder mice were originally purchased from the Jackson 
Laboratory (Bar Harbor, MA, USA), then bred on site. Epha4 knockout mice (allele name: Epha4tm1Byd) on a mixed C57BL/6, C57BL/10 and 129 genetic background, originally created by Dottori et al. [71], were generously donated by Prof. Elena Pasquale (Sanford Burnham Prebys, San Diego, CA, USA) then bred at UCSD. The mouse line C57BL/6 N-Dlgap1<em1(IMPC)Tcp> was made as part of the KOMP2Phase2 project at The Centre for Phenogenomics, Toronta, Canada. It was obtained from the Canadian Mouse Mutant Repository and bred at UCSD. Breeding from heterozygous parents produced, for Dlgap1, wild-type (WT), heterozygote (Het), and homozygote knockout (KO) offspring. For Epha4, homozygote knockout offspring usually died before weaning, leaving Het and WT offspring only. Within 3 days of weaning, we paired one focal FVB mouse with either a Dlgap1 (WT, Het, or KO) or an Epha4 (WT or Het) cage mate of the same sex (male or female). Immediately prior to pairing, the FVB mice were ear punched on each ear using 2$\mathrm{mm}$ ear punch scissors. Pairs of mice were then left to interact for 2 months before all mice were phenotyped in the forced swim test (FST) and sacrificed and the ears of FVB mice were collected. The sample size was 52 Epha4 Het mice and 33 Epha4 WT mice for wound healing; for FST, there were only 48 Epha4 Het mice as one mouse died during the FST, two mice had to be separated from their cage mate due to fighting in the days before the FST (but their ears were still collected as this did not significantly change the healing time), and during the FST of the fourth mouse, the battery of the camera ran out. A small subset of mice were video recorded in a new enclosure for $24 \mathrm{~h}$ a few days before the FST but the data from this pilot project are not reported here. Throughout the experiment, all mice were housed on a 12-h:12-h light-dark cycle, with lights on at 06:00, and all behavioral testing occurred during the light phase of the light-dark cycle.

\section{Forced swim test}

Following the same protocol as in the CFW study [36], mice were tested in the forced

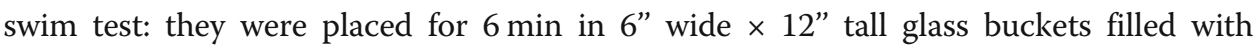
water at $24-26^{\circ} \mathrm{C}$. Mice were video recorded from the side and their immobility during the first 2 and last $4 \mathrm{~min}$ of the test was scored by an observer blind to the genotypes of the black (Epha4 and Dlgap1) mice. The analysis of IGE focused on immobility of FVB mice in the last 4 min of the test as FVB mice are rarely immobile during the first 2 min of the test.

\section{Healing from an ear punch}

Both ears of FVB mice were punched with a 2-mm-diameter ear punch scissor just before the mice were paired with an Epha4 or a Dlgap1 cage mate at weaning. Following the same protocol as in the CFW study [36], the ears were collected 2 months later after sacrifice and stored in $10 \%$ buffered formalin phosphate until analysis. To measure the area of the hole, each ear was mounted on a histology slide and photos were taken from a fixed distance. Images were analyzed with the ImageJ software [72] and the average across the two ears calculated. 


\section{Genotyping}

For genotyping Epha4 mice, tail or ear biopsies were sent to Transnetyx Inc. for genotyping (Transnetyx Genotyping Services, Cordova, TN). Transnetyx Inc. utilize realtime PCR and duplicate sample processing to ensure the accuracy of each mutation. Additionally, Sanger sequencing and gene expression analysis were performed to further validate the results of the Transnetyx assays.

For genotyping Dlgap1 mice, we used a multiplex PCR with primers: CCGTAAGTGA AGTCTCCATCAACAG (Fw1), CGGCTAGGATTTCAGAGTTTGTTC (Fw2) and CTTC CTCTCCTACACCATCAACAC (Rev1), yielding a 308-bp band in the presence of a WT allele and a 392-bp band in the presence of a knockout allele.

\section{Statistical analysis}

For both FST immobility and wound healing, five fixed effect models were first compared using the Akaike Information Criterion (AIC): a model with intercept only, a model with the sex of the pair (focal animal and cage mate were always of the same sex), a model with the genotype of the cage mate, a model with both sex and genotype of cage mate, and finally a model with main effects of sex and genotype of cage mate and their interaction.

IGE were then tested in males using an analysis of variance (ANOVA) with one degree of freedom.

\section{Supplementary Information}

The online version contains supplementary material available at https://doi.org/10.1186/s13059-021-02415-x.

Additional file 1: Table S1. Cages for all mice across their time at the phenotyping facility.

Additional file 2: Supplementary note. Correlation $\rho$ between direct and social genotypes arising from using each individual as both focal individual and social partner. Fig. S1. Estimation of the correlation $\rho$ between IGE and DGE in simulations. Fig. S2. Correlations between direct and social genotypes of CFW mice, and implications for GWAS. Fig. S3. Proportion of phenotypic variance explained by significant DGE and IGE loci. Fig. S4. Power to detect DGE and IGE associations in simulations. Fig. S5. Quantile-quantile (QQ) plot for the igeGWAS P values for Immobility during the first two minutes of the forced swim test. Fig. S6. Information relevant to the role of Dlgap1 in giving rise to IGE on immobility during the last four minutes of the FST. Fig. S7. Phenotypes of the outbred CFW mice used in igeGWAS.

Additional file 3: Table S2. Information about each phenotype, aggregate contributions of IGE and DGE, and correlation between IGE and DGE.

Additional file 4: Table S3. Genome-wide significant IGE associations (per-phenotype FDR $<10 \%$ ).

Additional file 5: Table S4. Genome-wide significant DGE associations (per-phenotype FDR $<10 \%$ ).

Additional file 6:. Review history.

\section{Acknowledgements}

The authors want to thank Drs. Na Cai, Robert W. Davies, and Richard Mott for facilitating access to the genotype data, and Dr. Elena Pasquale for generously providing mice from the EphA4 knockout line, which was produced thanks to funding from NIH grant NS087070. The authors also want to thank Dr. Jason Wolf and anonymous reviewers to their comments, which contributed to improving the manuscript.

\section{Peer review information}

Barbara Cheifet was the primary editor of this article and managed its editorial process and peer review in collaboration with the rest of the editorial team.

\section{Review history}

The review history is available as Additional file 6.

\section{Authors' contributions}

$A B, A A P$, and $O S$ designed the study. $A B$ and FPC performed the analyses. JN contributed the cage information. $A B$, $A B L, N F, C M$, and $B Y$ performed the mouse knockout experiments. All authors contributed to the interpretation of the data. AB, FPC, AAP, and OS wrote the manuscript. The authors read and approved the final manuscript. 
Authors' information

Twitter handles: @AmelieBaud (Amelie Baud); @YalcinBinnaz (Binnaz Yalcin); @Jerome_Nicod (Jerome Nicod); @AbePalmer (Abraham A. Palmer); @OliverStegle (Oliver Stegle).

\section{Funding}

AB was supported by a fellowship from the Wellcome Trust (105941/Z/14/Z). This work was partially supported by a pilot grant from NIH (P50DA037844 to AAP). Research in the Stegle lab is supported by core funding from EMBL, the BMBF, the Volkswagen Foundation, and the EU (ERC project DECODE 810296). The funders had no role in the design of the study, analysis and interpretation of the results, nor in writing the manuscript. Open Access funding enabled and organized by Projekt DEAL.

\section{Availability of data and materials}

Genotype and phenotype data from Nicod et al. [36] and Davies et al. [37] are available from http://wp.cs.ucl.ac.uk/ outbredmice/. Cage information is provided in Additional file 1: Table S1. CFW hippocampus data is available in GeneNetwork (https://genenetwork.org) as FAIR data and can be analysed reproducibly using the online GWAS webservice. GeneNetwork contains over 20-years of experimental mouse and rat data.

The code used for variance decomposition and GWAS in this study is available from https://github.com/Baud-lab under an Apache 2.0 license and from zenodo $[73,74]$.

\section{Declarations}

\section{Ethics approval}

All animal procedures were approved by the Institutional Animal Care and Use Committee of the University of California San Diego (UCSD) and were conducted in accordance with the NIH Guide for the Care and Use of Laboratory Animals.

\section{Consent for publication}

Not applicable

\section{Competing interests}

The authors declare that they have no competing interests.

\section{Author details}

${ }^{1}$ European Molecular Biology Laboratory, European Bioinformatics Institute, Wellcome Genome Campus, CB10 1SD Hinxton, Cambridge, UK. ${ }^{2}$ Department of Psychiatry, University of California San Diego, La Jolla, CA 92093, USA. ${ }^{3}$ Current Address: Centre for Genomic Regulation (CRG), The Barcelona Institute of Science and Technology, Dr. Aiguader 88, 08003 Barcelona, Spain. ${ }^{4}$ Microsoft Research New England, Cambridge, MA, USA. ${ }^{5}$ INSERM U1231 GAD Laboratory, University Bourgogne Franche-Comté, 21070 Dijon, France. ${ }^{6}$ Wellcome Centre for Human Genetics, University of Oxford, Oxford, UK. ${ }^{7}$ Current Address: The Francis Crick Institute, London, UK. ${ }^{8}$ Institute for Genomic

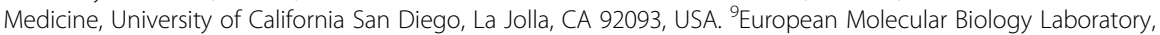
Genome Biology Unit, Heidelberg, Germany. ${ }^{10}$ Division of Computational Genomics and Systems Genetics, German Cancer Research Center, 69120 Heidelberg, Germany. ${ }^{11}$ Wellcome Sanger Institute, Wellcome Genome Campus, CB10 1SD Hinxton, Cambridge, UK.

Received: 27 April 2021 Accepted: 21 June 2021

Published online: 26 July 2021

\section{References}

1. Griffing B. Selection in reference to biological groups I. Individual and group selection applied to populations of unordered groups. Aust J Biol Sci. 1967;20(1):127-40. https://doi.org/10.1071/B|9670127.

2. Moore AJ, Brodie ED III, Wolf JB. Interacting phenotypes and the evolutionary process: I. Direct and indirect genetic effects of social interactions. Evolution. 1997;51(5):1352-62. https://doi.org/10.1111/j.1558-5646.1997.tb01458.x.

3. Wolf JB, Brodie ED III, Cheverud JM, Moore AJ, Wade MJ. Evolutionary consequences of indirect genetic effects. Trends Ecol Evol. 1998;13(2):64-9. https://doi.org/10.1016/S0169-5347(97)01233-0.

4. Wolf JB, Mutic JJ, Kover PX. Functional genetics of intraspecific ecological interactions in Arabidopsis thaliana. Philos Transact R Soc B Biol Sci. 2011;366(1569):1358-67. https://doi.org/10.1098/rstb.2010.0239.

5. Bailey NW, Hoskins JL. Detecting cryptic indirect genetic effects. Evolution. 2014;68(7):1871-82. https://doi.org/10.1111/ evo.12401.

6. Bleakley $\mathrm{BH}$, Brodie ED. Indirect genetic effects influence antipredator behavior in guppies: estimates of the coefficient of interaction psi and the inheritance of reciprocity. Evolution. 2009;63(7):1796-806. https://doi.org/10.1111/j.1558-5646.2 009.00672.x.

7. Ashbrook DG, Gini B, Hager R. Genetic variation in offspring indirectly influences the quality of maternal behaviour in mice. Elife. 2015:4:11814.

8. Ashbrook DG, Sharmin N, Hager R, editors. Offspring genes indirectly influence sibling and maternal behavioural strategies over resource share. Proc R Soc B. 2017;284:20171059.

9. Baud A, Mulligan MK, Casale FP, Ingels JF, Bohl CJ, Callebert J, et al. Genetic variation in the social environment contributes to health and disease. Plos Genet. 2017;13(1):e1006498. https://doi.org/10.1371/journal.pgen.1006498.

10. Head ML, Berry LK, Royle NJ, Moore AJ. Paternal care: direct and indirect genetic effects of fathers on offspring performance. Evolution. 2012;66(11):3570-81. https://doi.org/10.1111/j.1558-5646.2012.01699.x.

11. Mutic JJ, Wolf JB. Indirect genetic effects from ecological interactions in Arabidopsis thaliana. Mol Ecol. 2007;16(11): 2371-81. https://doi.org/10.1111/j.1365-294X.2007.03259.x. 
12. Petfield D, Chenoweth SF, Rundle HD, Blows MW. Genetic variance in female condition predicts indirect genetic variance in male sexual display traits. Proc Natl Acad Sci. 2005;102(17):6045-50. https://doi.org/10.1073/pnas.0409378102.

13. Wilson AJ, Gelin U, Perron M-C, Réale D. Indirect genetic effects and the evolution of aggression in a vertebrate system. Proc R Soc London B Biol Sci. 2009;276(1656):533-41.

14. Moore AJ, Haynes KF, Preziosi RF, Moore PJ. The evolution of interacting phenotypes: genetics and evolution of social dominance. Am Nat. 2002;160(S6):S186-S97.

15. Bergsma R, Kanis E, Knol EF, Bijma P. The contribution of social effects to heritable variation in finishing traits of domestic pigs (Sus scrofa). Genetics. 2008;178(3):1559-70. https://doi.org/10.1534/genetics.107.084236.

16. Ellen ED, Visscher J, van Arendonk JA, Bijma P. Survival of laying hens: genetic parameters for direct and associative effects in three purebred layer lines. Poultry Sci. 2008;87(2):233-9. https://doi.org/10.3382/ps.2007-00374.

17. Alemu SW, Bijma P, Møller SH, Janss L, Berg P. Indirect genetic effects contribute substantially to heritable variation in aggression-related traits in group-housed mink (Neovison vison). Genet Select Evol. 2014;46(1):30. https://doi.org/10.11 86/1297-9686-46-30.

18. e Silva JC, Potts B, Gilmour A, Kerr R. Genetic-based interactions among tree neighbors: identification of the most influential neighbors, and estimation of correlations among direct and indirect genetic effects for leaf disease and growth in Eucalyptus globulus. Heredity. 2017;119(3):125.

19. Wilson A, Coltman D, Pemberton J, Overall A, Byrne K, Kruuk L. Maternal genetic effects set the potential for evolution in a free-living vertebrate population. J Evol Biol. 2005;18(2):405-14. https://doi.org/10.1111/j.1420-9101.2 004.00824.X.

20. McAdam AG, Boutin S, Réale D, Berteaux D. Maternal effects and the potential for evolution in a natural population of animals. Evolution. 2002;56(4):846-51. https://doi.org/10.1111/j.0014-3820.2002.tb01396.x.

21. Hadfield JD, Burgess MD, Lord A, Phillimore AB, Clegg SM, Owens IP. Direct versus indirect sexual selection: genetic basis of colour, size and recruitment in a wild bird. Proc R Soc B Biol Sci. 2006;273(1592):1347-53. https://doi.org/10.1 098/rspb.2005.3459.

22. Kong A, Thorleifsson G, Frigge ML, Vilhjalmsson BJ, Young Al, Thorgeirsson TE, et al. The nature of nurture: effects of parental genotypes. Science. 2018;359(6374):424-8. https://doi.org/10.1126/science.aan6877.

23. Bates TC, Maher BS, Medland SE, McAloney K, Wright MJ, Hansell NK, et al. The nature of nurture: using a virtual-parent design to test parenting effects on children's educational attainment in genotyped families. Twin Res Hum Genet. 2018:1-11.

24. Warrington NM, Beaumont RN, Horikoshi M, Day FR, Helgeland $\varnothing$, Laurin C, et al. Maternal and fetal genetic effects on birth weight and their relevance to cardio-metabolic risk factors. Nature genetics. 2019;51(5):804-14.

25. Sotoudeh R, Harris KM, Conley D. Effects of the peer metagenomic environment on smoking behavior. Proc Natl Acad Sci. 2019;116(33):16302-7. https://doi.org/10.1073/pnas.1806901116.

26. Xia C, Canela-Xandri O, Rawlik K, Tenesa A. Evidence of horizontal indirect genetic effects in humans. Nat Hum Behav. 2021;5(3):399-406. https://doi.org/10.1038/s41562-020-00991-9.

27. Demange PA, Hottenga JJ, Abdellaoui A, Eilertsen EM, Malanchini M, Domingue BW, et al. Estimating effects of parents' cognitive and non-cognitive skills on offspring education using polygenic scores. bioRxiv. 2021:2020.09.15.296236.

28. McGlothlin JW, Brodie ED III. How to measure indirect genetic effects: the congruence of trait-based and variancepartitioning approaches. Evolution. 2009;63(7):1785-95. https://doi.org/10.1111/j.1558-5646.2009.00676.x.

29. Bijma P. The quantitative genetics of indirect genetic effects: a selective review of modelling issues. Heredity. 2014; 112(1):61-9. https://doi.org/10.1038/hdy.2013.15.

30. Wilson AJ, Morrissey M, Adams M, Walling CA, Guinness FE, Pemberton JM, et al. Indirect genetics effects and evolutionary constraint: an analysis of social dominance in red deer, Cervus elaphus. J Evol Biol. 2011;24(4):772-83. https://doi.org/10.1111/j.1420-9101.2010.02212.x.

31. Brinker T, Bijma P, Vereijken A, Ellen ED. The genetic architecture of socially-affected traits: a GWAS for direct and indirect genetic effects on survival time in laying hens showing cannibalism. Genet Select Evol. 2018;50(1):38. https:// doi.org/10.1186/s12711-018-0409-7.

32. Wu P, Wang K, Yang Q, Zhou J, Chen D, Liu Y, et al. Whole-genome re-sequencing association study for direct genetic effects and social genetic effects of six growth traits in Large White pigs. Sci Rep. 2019;9(1):1-12.

33. Hong J-K, Lee J-B, Ramayo-Caldas Y, Kim S-D, Cho E-S, Kim Y-S, et al. Single-step genome-wide association study for social genetic effects and direct genetic effects on growth in Landrace pigs. Sci Rep. 2020;10(1):1-11.

34. Warrington NM, Beaumont RN, Horikoshi M, Day FR, Helgeland $\varnothing$, Laurin C, et al. Maternal and fetal genetic effects on birth weight and their relevance to cardio-metabolic risk factors. Nat Genet. 2019;51(5):804-14. https://doi.org/10.1038/ s41588-019-0403-1.

35. McGinnis R, Steinthorsdottir V, Williams NO, Thorleifsson G, Shooter S, Hjartardottir S, et al. Variants in the fetal genome near FLT1 are associated with risk of preeclampsia. Nat Genet. 2017:49(8):1255-60. https://doi.org/10.1038/ng.3895.

36. Nicod J, Davies RW, Cai N, Hassett C, Goodstadt L, Cosgrove C, et al. Genome-wide association of multiple complex traits in outbred mice by ultra-low-coverage sequencing. Nat Genet. 2016;48(8):912-8. https://doi.org/10.1038/ng.3595.

37. Davies RW, Flint J, Myers S, Mott R. Rapid genotype imputation from sequence without reference panels. Nat Genet. 2016:48(8):965-9. https://doi.org/10.1038/ng.3594.

38. Sadler AM, Bailey SJ. Repeated daily restraint stress induces adaptive behavioural changes in both adult and juvenile mice. Physiol Behav. 2016;167:313-23. https://doi.org/10.1016/j.physbeh.2016.09.014.

39. Biscarini F, Bovenhuis H, Van Der Poel J, Rodenburg T, Jungerius A, Van Arendonk J. Across-line SNP association study for direct and associative effects on feather damage in laying hens. Behav Genet. 2010;40(5):715-27. https://doi.org/10.1 007/s10519-010-9370-0.

40. Casale FP, Rakitsch B, Lippert C, Stegle O. Efficient set tests for the genetic analysis of correlated traits. Nat Methods. 2015;12(8):755-8. https://doi.org/10.1038/nmeth.3439.

41. Sudmant PH, Rausch T, Gardner EJ, Handsaker RE, Abyzov A, Huddleston J, et al. An integrated map of structural variation in 2,504 human genomes. Nature. 2015:526(7571):75-81. https://doi.org/10.1038/nature15394.

42. Beavis W. QTL analyses: power, precision and accuracy. Molecular Dissection of Complex Traits. Edited by: AH P. 1997. CRC Press, New York.

43. Beavis W, Beavis W. The power and deceit of QTL experiments: lessons from comparative QTL studies; 1994. 
44. Yalcin B, Nicod J, Bhomra A, Davidson S, Cleak J, Farinelli L, et al. Commercially available outbred mice for genome-wide association studies. Plos Genet. 2010;6(9):e1001085. https://doi.org/10.1371/journal.pgen.1001085.

45. Parker CC, Gopalakrishnan S, Carbonetto P, Gonzales NM, Leung E, Park YJ, et al. Genome-wide association study of behavioral, physiological and gene expression traits in outbred CFW mice. Nat Genet. 2016;48(8):919-26. https://doi. org/10.1038/ng.3609.

46. Amelie B. locusZooms_TableS3.zip2021.

47. Commons KG, Cholanians AB, Babb JA, Ehlinger DG. The rodent forced swim test measures stress-coping strategy, not depression-like behavior. ACS Chem Neurosci. 2017;8(5):955-60. https://doi.org/10.1021/acschemneuro.7b00042.

48. Lupiáñez DG, Kraft K, Heinrich V, Krawitz P, Brancati F, Klopocki E, et al. Disruptions of topological chromatin domains cause pathogenic rewiring of gene-enhancer interactions. Cell. 2015;161(5):1012-25. https://doi.org/10.1016/j.cell.2015. 04.004.

49. Dixon JR, Selvaraj S, Yue F, Kim A, Li Y, Shen Y, et al. Topological domains in mammalian genomes identified by analysis of chromatin interactions. Nature. 2012;485(7398):376-80. https://doi.org/10.1038/nature11082.

50. Bouvier D, Corera AT, Tremblay MË, Riad M, Chagnon M, Murai KK, et al. Pre-synaptic and post-synaptic localization of EphA4 and EphB2 in adult mouse forebrain. J Neurochem. 2008;106(2):682-95. https://doi.org/10.1111/j.1471-4159.2008. 05416.x.

51. Murai KK, Nguyen LN, Irie F, Yamaguchi Y, Pasquale EB. Control of hippocampal dendritic spine morphology through ephrin-A3/EphA4 signaling. Nat Neurosci. 2003;6(2):153-60. https://doi.org/10.1038/nn994.

52. Li Y, Wang H, Wang X, Liu Z, Wan Q, Wang G. Differential expression of hippocampal EphA4 and ephrinA3 in anhedonic-like behavior, stress resilience, and antidepressant drug treatment after chronic unpredicted mild stress. Neurosci Lett. 2014;566:292-7. https://doi.org/10.1016/j.neulet.2014.03.008.

53. J-c Z, Yao W, Qu Y, Nakamura M, Dong C, Yang C, et al. Increased EphA4-ephexin1 signaling in the medial prefrontal cortex plays a role in depression-like phenotype. Sci Rep. 2017;7(1):7133.

54. Coba M, Ramaker M, Ho E, Thompson S, Komiyama N, Grant S, et al. Dlgap1 knockout mice exhibit alterations of the postsynaptic density and selective reductions in sociability. Sci Rep. 2018;8(1):1-12.

55. de Oliveira PC, Zaniboni CR, Carmona IM, Fonseca AR, Canto-de-Souza A. Preliminary behavioral assessment of cagemates living with conspecifics submitted to chronic restraint stress in mice. Neurosci Lett. 2017;657:204-10. https:// doi.org/10.1016/j.neulet.2017.07.008.

56. Bolger N, DeLongis A, Kessler RC, Wethington E. The contagion of stress across multiple roles. J Marriage Fam. 1989; 51(1):175-83. https://doi.org/10.2307/352378.

57. Haeffel GJ, Hames JL. Cognitive vulnerability to depression can be contagious. Clin Psychol Sci. 2014;2(1):75-85. https:// doi.org/10.1177/2167702613485075.

58. Stickney JD, Morgan MM. Social housing promotes recovery of wheel running depressed by inflammatory pain and morphine withdrawal in male rats. Behav Brain Res. 2021;396:112912. https://doi.org/10.1016/j.bbr.2020.112912.

59. Clarke T-K, Adams MJ, Howard DM, Xia C, Davies G, Hayward C, et al. Genetic and shared couple environmental contributions to smoking and alcohol use in the UK population. Mol Psychiatry. 2019:1-11.

60. Ridaura VK, Faith JJ, Rey FE, Cheng J, Duncan AE, Kau AL, et al. Gut microbiota from twins discordant for obesity modulate metabolism in mice. Science. 2013;341(6150):1241214. https://doi.org/10.1126/science.1241214.

61. Sittig LJ, Carbonetto P, Engel KA, Krauss KS, Barrios-Camacho CM, Palmer AA. Genetic background limits generalizability of genotype-phenotype relationships. Neuron. 2016;91(6):1253-9. https://doi.org/10.1016/j.neuron.2016.08.013.

62. Prevost N, Woulfe D, Tanaka T, Brass LF. Interactions between Eph kinases and ephrins provide a mechanism to support platelet aggregation once cell-to-cell contact has occurred. Proc Natl Acad Sci. 2002;99(14):9219-24. https://doi.org/10.1 073/pnas.142053899.

63. Doku HC. The thromboplastic activity of human saliva. J Dent Res. 1960;39(6):1210-21. https://doi.org/10.1177/0022034 5600390061501.

64. Brand HS, Ligtenberg AJ, Veerman EC. Saliva and wound healing. Saliva. 2014;24:52-60.

65. Venerables W, Ripley B. Modern applied statistics with S. New York: Springer; 2002.

66. Bijma P, Muir WM, Ellen ED, Wolf JB, Van Arendonk JA. Multilevel selection 2: estimating the genetic parameters determining inheritance and response to selection. Genetics. 2007;175(1):289-99. https://doi.org/10.1534/genetics.106. 062729.

67. Dabney A, Storey JD, Warnes G. qvalue: Q-value estimation for false discovery rate control. R package version. 2010;1(0).

68. Pruim RJ, Welch RP, Sanna S, Teslovich TM, Chines PS, Gliedt TP, et al. LocusZoom: regional visualization of genomewide association scan results. Bioinformatics. 2010;26(18):2336-7. https://doi.org/10.1093/bioinformatics/btq419.

69. Mulligan MK, Mozhui K, Prins P, Williams RW. GeneNetwork: a toolbox for systems genetics. Systems Genetics: Springer; 2017. p. $75-120$

70. Sloan Z, Arends D, Broman KW, Centeno A, Furlotte N, Nijveen H, et al. GeneNetwork: framework for web-based genetics. J Open Source Softw. 2016;1(2)

71. Dottori M, Hartley L, Galea M, Paxinos G, Polizzotto M, Kilpatrick T, et al. EphA4 (Sek1) receptor tyrosine kinase is required for the development of the corticospinal tract. Proc Natl Acad Sci. 1998;95(22):13248-53. https://doi.org/10.1 073/pnas.95.22.13248.

72. Schneider CA, Rasband WS, Eliceiri KW. NIH Image to ImageJ: 25 years of image analysis. Nat Methods. 2012;9(7):671-5. https://doi.org/10.1038/nmeth.2089.

73. Amelie Baud FPC. https://github.com/Baud-lab/CFW. v1.0 ed. GitHub2021. Accessed 16 June 2021.

74. Amelie Baud FPC. https://github.com/Baud-lab/core_VD. v1.0 ed. GitHub2021. Accessed 16 June 2021.

\section{Publisher's Note}

Springer Nature remains neutral with regard to jurisdictional claims in published maps and institutional affiliations. 